\title{
Community asthma clinics: 1993 survey of primary care by the National Asthma Task Force
}

G Barnes, M R Partridge, on behalf of the Organisation of Care Working Group of the National Asthma Task Force

\begin{abstract}
Objectives-To establish a baseline of work done in primary care asthma clinics in the United Kingdom and to assess the degree of clinical delegation to nurses and the appropriateness of their training.

Design-Prospective questionnaire survey of asthma care in general practices and a subsidiary survey of all family health services authorities (FHSAs) of the number of asthma clinics in their area.
\end{abstract}

Setting-All 14251 general practices in the United Kingdom and 117 FHSAs or health boards (Scotland and Northern Ireland).

Results-Questionnaires were returned by $4327(30.4 \%)$ general practices, 54\% being completed by practice nurses and $22 \%$ by general practitioners; in $24 \%$ profession was not stated. In all, $77 \cdot 2 \%$ (3339/4327) of respondents ran an asthma clinic. 60 FHSAs stated the number of asthma clinics at the time of the general practice survey (total 3653 clinics); within responding FHSAs $1702(46 \cdot 6 \%)$ practices running an asthma clinic replied to the general practice survey. Clinics exclusive for patients with asthma mostly occurred in practices with five or more general practitioners $(70.2 \%)$, compared with singlehanded practices $(31 \cdot 7 \%)$. The average number of asthma clinics run per practice was five a month; the average duration was 2 hours and 20 minutes. $1131(48 \cdot 8 \%)$ nurses ran clinics by themselves, 1180 $(47.9 \%)$ with the doctor, and $39(1 \cdot 7 \%)$ had no medical input. Comprehensive questioning occurred other than for nasal $(872,26 \cdot 1 \%)$ or oesophageal $(335,10 \cdot 0 \%)$ symptoms and use of aspirin and nonsteroidal drugs $(1161,33 \cdot 4 \%)$. Growth in children was measured by only a third of respondents. Of the 1131 nurses who ran clinics alone, $251(22 \cdot 2 \%)$ did so without formal training entailing assessment.

Conclusion-Asthma clinics are now common in general practice and much of their work is done by nurses, a significant minority of whom may not have had sufficient training.

Implications-As this survey is probably biased toward the more asthma aware practices, greater deficiencies in training and standards may exist in other practices. Further evaluation of the effectiveness of asthma clinics is needed. (Quality in Health Care 1994;3:133-136)
Introduction

An estimated three million people in the United Kingdom have asthma, ${ }^{1}$ a condition for which most care is appropriately provided in the community. The organisation of that care varies from practice to practice, but an increasing number of general practitioners have established asthma clinics, which enable care for those with chronic asthma to be centred on regular review rather than by managing crises. As a result of the government's encouragement in the form of financial support for health promotion clinics (and from July 1993 the chronic disease management scheme) there has been an increased trend towards sharing the care of longer term conditions with practice nurses.

However, little is known nationally about how asthma care is organised within primary care. This survey was undertaken to determine (a) the activities taking place within primary care asthma clinics and (b) the training provided for nurses involved in running asthma clinics. The survey should provide a baseline for future comparisons after introduction of the 1993 Chronic Disease Management Regulations for Asthma in General Practice.

\section{Methods}

SURVEY OF GENERAL PRACTICES

A postal questionnaire was sent to each general practice in the United Kingdom, a total of 14251 questionnaires. They were addressed personally to the practice nurses when their name was known, or otherwise to the "practice nurse or the general practitioner with an interest in asthma." A covering letter from the two principal authors (GB, MRP) outlined the aims of the survey.

The questionnaire was devised by the National Asthma Task Force; after pilot studies among practice nurses and general practitioners it contained 46 questions designed to elicit information about the following:

(a) Practice details (number of doctors, number of patients, number on asthma register, and whether an asthma clinic was established)

(b) Asthma clinic details (number of clinics held, number of patients attending, details of protocols, resources available, activities undertaken)

(c) Staff training and external relationships.

SURVEY OF FAMILY HEALTH SERVICES

AUTHORITIES (FHSAS)

The questionnaires sent to general practitioners were identified only by FHSAs. After 
the main survey of general practices was complete a letter was sent to all FHSAs (or health boards in Scotland and Northern Ireland) requesting information about the number of asthma clinics known by them to have been operating at the time of that survey (March 1993). For the FHSAs who responded the proportion of practices running asthma clinics who had replied to the general practice questionnaire could be determined.

\section{Results}

Questionnaires were returned by 4327 practices, a $30.4 \%$ response rate. In all, $54 \%$ had been completed by the practice nurse and $22 \%$ by a general practitioner, with $24 \%$ not stating their profession. Of the 4327 respondents, $3339(77 \cdot 2 \%)$ ran some form of asthma clinic.

Sixty of 117 FHSAs (or health boards) responded to our request for information about the number of asthma clinics being run in their area at the time of the primary care survey, and they reported 3653 clinics being in operation. Analysing practice responses only from practices in FHSAs who responded shows that a reply to the main questionnaire was received from 1702 practices running an asthma clinic out of an apparent possible total of 3653, giving a $46 \cdot 6 \%$ response rate by practices running asthma clinics.

\section{CHARACTERISTICS OF RESPONDENTS}

Responding practices had an average of four general practitioners and an average practice list size of 6838 . Most practices with five general practitioners or more ran an exclusive clinic for patients with asthma $(70 \cdot 2 \%)$ whereas only $31 \cdot 7 \%$ of singlehanded general practitioners did so (table 1).

\section{ORGANISATION OF ASTHMA CLINICS}

Overall, 2366 respondents $(54 \cdot 7 \%)$ ran clinics exclusively for patients with asthma, 567 $(13.1 \%)$ ran mixed clinics which asthmatic patients attended, and $406(9 \cdot 4 \%)$ had both exclusive and mixed clinics. The number of exclusive asthma clinics run by each practice was proportional to its practice size. Overall, the average number of asthma clinics run per practice was five a month. The average length of an asthma clinic was two hours and 20 minutes (range 51-200 minutes), and the average number of asthmatic patients attending clinics run exclusively for them was 35 a month. In all, $2152(90.9 \%)$ of the 2366 practices running exclusive asthma clinics had an asthma register (compared with 3552 $(77 \%)$ of all respondents). Of the 3339 respondents who ran asthma clinics, 2072 $(62 \cdot 1 \%)$ saw patients in their clinics by syste- matic recall from the asthma register. Only 175 $(5 \cdot 2 \%)$ said that these clinics were run by general practitioners alone, with (2248) $(67 \cdot 3 \%)$ being reported as run by a general practitioner and nurse and $866(25.9 \%)$ by a nurse alone.

\section{CLINIC ACTIVITIES}

The mean time reported as spent with each patient at the initial assessment or consultation was 22 minutes, with that in follow up clinics averaging 13 minutes. Two thirds of clinics reported having peak flow meters to loan, and 878 practices $(26 \cdot 2 \%)$ had spirometers, although over a quarter of these reported that they did not use them. Placebo inhaler devices of all the common types were widely available. In the asthma clinics respondents $(n=3339)$ reported a high level of questioning of patients about their family history $(2925,87 \cdot 6 \%)$, occupational history $(2282,68 \cdot 3 \%)$, evidence of atopy $(2800,83.9 \%)$, and trigger factors $(2931,87 \cdot 8 \%)$, and almost universal inquiry regarding symptoms $(2997,89 \cdot 8 \%)$, smoking $(3135,93.9 \%)$, treatment $(3204,96.0 \%)$, inhaler technique $(3031,90 \cdot 8 \%)$, and asthma attacks $(2619,78 \cdot 4 \%)$. However, routine inquiry about patients' use of aspirin and nonsteroidal drugs $(1161,33.4 \%$ ) or about nasal $(872,26 \cdot 1 \%)$ or oesophageal symptoms (335, $10.0 \%$ ) were uncommon. The proportion of practices indicating that they always inquired about days lost from school or work was low compared with other factors $(2058,61 \cdot 6 \%)$. Most $(2720,81.5 \%)$ of all those running asthma clinics indicated that they always worked out predicted levels of peak flow at the initial clinic assessment, $340(10 \cdot 2 \%)$ often did so, $133(4.0 \%)$ sometimes did so, and 44 $(1 \cdot 3 \%)$ said that they never did so.

Table 2 shows the activities during follow up clinic visits according to the frequency of the activities. Monitoring the growth of children seemed to be an uncommon practice as only a third of respondents always did this.

\section{PATIENT EDUCATION}

Most of the 3339 respondents in practices which ran asthma clinics or mixed clinics said that it was always their practice to spend time educating patients about asthma at the initial consultation $2558(76.6 \%)$, teaching them to recognise signs of worsening asthma (2246, $67 \cdot 3 \%$ ), checking inhaler technique 2693, $80.7 \%$ and explaining about preventers and relievers $(2246,76 \cdot 8 \%)$. It was much less likely that those running the clinic would always explore the patient's or the family's perceptions of asthma $(1147,34.4 \%)$, or always allow the patient to choose their inhaler device $(822$,

Table 1 Types of clinic run, by practice size (number of GPs). Figures are numbers (percentages)

\begin{tabular}{lcccccc}
\hline & All practices & \multicolumn{4}{c}{ No of GPs in practice } \\
\cline { 3 - 6 } & & 1 & 2 & 3 & 4 & $\geqslant 5$ \\
\hline Clinic exclusively for asthmatic patients & $2366(54 \cdot 7)$ & $216(31 \cdot 7)$ & $328(42 \cdot 2)$ & $417(51 \cdot 8)$ & $518(64 \cdot 8)$ & $876(70 \cdot 2)$ \\
Mixed clinic which asthmatic patients attend & $567(13 \cdot 1)$ & $148(21 \cdot 7)$ & $125(16 \cdot 1)$ & $104(12 \cdot 9)$ & $71(8 \cdot 8)$ & $117(9 \cdot 3)$ \\
Both exclusive and mixed clinics & $406(9 \cdot 4)$ & $79(11 \cdot 6)$ & $95(12 \cdot 2)$ & $79(9 \cdot 8)$ & $62(7 \cdot 7)$ & $89(7 \cdot 1)$ \\
No clinics for asthmatic patients & $988(22 \cdot 8)$ & $238(34 \cdot 9)$ & $229(29 \cdot 4)$ & $204(25 \cdot 4)$ & $148(18 \cdot 5)$ & $165(13 \cdot 2)$ \\
\hline Total & 4327 & 681 & 777 & 804 & 799 & 1247 \\
\hline
\end{tabular}


Table 2 Frequency of inquiry or action during follow up clinic visit for exclusive or mixed clinics, or both (3339 respondents). Figures are numbers (percentages)

\begin{tabular}{|c|c|c|c|c|c|}
\hline Inquiry or action & Always & Often & Sometimes & Never & $N S$ \\
\hline Days lost from school or work & $1975(59 \cdot 1)$ & $866(25 \cdot 9)$ & $363(10 \cdot 9)$ & $35(1 \cdot 0)$ & $100(3)$ \\
\hline Being woken at night & $2924(87 \cdot 5)$ & $289(8 \cdot 4)$ & $32(1 \cdot 0)$ & $3(<1)$ & $91(2 \cdot 7)$ \\
\hline Restrictions going about daily activities & $2667(79 \cdot 9)$ & $493(14 \cdot 8)$ & $80(2 \cdot 4)$ & $7(<1)$ & $92(2.7)$ \\
\hline Frequency of use of short acting bronchodilators & $3007(90 \cdot 0)$ & $213(6 \cdot 4)$ & $19(<1)$ & $2(<1)$ & $98(2 \cdot 9)$ \\
\hline Compliance with treatment & $3020(90 \cdot 5)$ & $205(6 \cdot 1)$ & $11(<1)$ & $2(<1)$ & $101(3.0)$ \\
\hline Check peak flow records & $2581(77 \cdot 3)$ & $547(16 \cdot 4)$ & $110(3 \cdot 3)$ & $8(<1)$ & $93(2 \cdot 8)$ \\
\hline Record comments on likely compliance in patients' notes & $2138(64 \cdot 0)$ & $746(22 \cdot 3)$ & $314(9 \cdot 4)$ & $37(1 \cdot 1)$ & $104(3 \cdot 1)$ \\
\hline Monitor growth in children & $1138(34 \cdot 1)$ & $1118(33 \cdot 5)$ & $736(22 \cdot 0)$ & $211(6 \cdot 3)$ & $136(4 \cdot 0)$ \\
\hline
\end{tabular}

Monitor growth in children

NS $=$ not stated

$24 \cdot 6 \%$ ), although $1211(36 \cdot 3 \%)$ allowed this often. A total of $2222(66 \cdot 5 \%)$ respondents usually provided a framework for a guided self management plan and $774(23 \cdot 2 \%)$ sometimes did so: $1420(42 \cdot 5 \%)$ wrote them themselves, $741(22 \cdot 2 \%)$ used National Asthma Campaign cards, and $675(20 \cdot 2 \%)$ used a preprinted practice self management plan. When such plans were provided by the practice nurse this related closely to the nurse's overall role in the clinic and to the training received.

EXTERNAL RELATIONS: INTERFACE BETWEEN PRIMARY AND SECONDARY CARE

Most of the responding practices reported limited contacts between primary and secondary care. A total of $2732(81 \cdot 8 \%)$ of those running clinics stated that there was limited referral of patients with asthma from practice to hospital, with only $142(4.3 \%)$ referring frequently; $2051(61 \cdot 4 \%)$ said there was limited communication between the practice and the hospital.

ROLE AND TRAINING OF PRACTICE NURSE Of the 3339 questionnaires returned from practices running asthma clinics or mixed clinics, $2317(69.4 \%)$ were completed by practice nurses and $917(27 \cdot 5 \%)$ by general practitioners. A total of $1131(48 \cdot 8 \%)$ practice nurses ran their clinics on their own (maximum role), $1180(47 \cdot 9 \%)$ shared patient care with a general practitioner (medium role), and 39 $(1.7 \%)$ of respondents had a minimum role (the patient always saw the general practitioner). The remainder did not specify. Of the nurses who had a maximum role, 695 $(61.5 \%)$ said that general practitioners were always available to offer advice, $322(28 \cdot 5 \%)$ that they were often available, $94(8 \cdot 3 \%)$ sometimes available, and $13(1 \cdot 2 \%)$ reported that they were never available. Of the practice nurses who ran clinics on their own, most (880, $77 \cdot 8 \%$ ) had received both advanced asthma training and attended other study days and workshops closely linked with the pharmaceutical industry (table 3). However, 251 $(22 \cdot 2 \%)$ of the practice nurses with a maximum role in asthma clinics who responded to this survey had not undertaken any evaluable advanced training.

\section{Discussion}

This survey was designed to give an insight into the operation of asthma clinics in primary care in the United Kingdom in 1993. The way the questionnaires were addressed is likely to have produced the observed bias towards respondents being more likely to be practice nurses. In view of the total number of practices and in order to maintain respondents' anonymity, we decided not to attempt to follow up non-responders. Practitioners working in primary care receive an appreciable amount of post, and the questionnaire used in this survey was lengthy and comprehensive. A response rate of $30 \%$, although low, was therefore not unexpected. The subsidiary FHSA survey suggests that those who completed our questionnaire were disproportionately likely to be running asthma clinics ( $46 \%$ of known asthma clinics were in responding FHSAs) and this needs to be borne in mind in interpreting the results. The survey gives an insight into the activities of an extremely large number of clinics; however, asthma care is probably less well organised by those who did not respond.

Larger practices were more likely than singlehanded practices to run asthma clinics, and the number of clinics run and patients seen was proportional to practice size. All seemed to have the necessary basic equipment to teach patients the necessary skills for monitoring and treating their condition, and the respondents claimed to ask patients routinely about aspects of care traditionally taught to general practitioners and practice nurses. However, given that this survey is probably biased towards the practices that are more aware of asthma, additional subjects are indicated which need to be incorporated in the future training of health professionals. More attention may need to be paid to the patient's occupation, their use of other drugs, their nasal and oesophageal symptoms, and to elicit patients' and families' feelings about having asthma. Of particular concern is the apparent underuse by nurses of measurements of growth during routine

Table 3 Asthma training received by practice nurse respondents. Figures are numbers (percentages). (Totals may exceed $100 \%$ because of training received from more than one source)

\begin{tabular}{lccc}
\hline & $\begin{array}{c}\text { Minimum role } \\
(n=39)\end{array}$ & $\begin{array}{c}\text { Medium role } \\
(n=1110)\end{array}$ & $\begin{array}{c}\text { Maximum role } \\
(n=1131)\end{array}$ \\
\hline Formal study days or workshops set up by pharmaceutical companies & $27(69 \cdot 2)$ & $891(80 \cdot 3)$ & $939(83 \cdot 0)$ \\
Formal study days or workshops set up by FHSA or health board & $21(53 \cdot 8)$ & $734(66 \cdot 1)$ & $721(63 \cdot 7)$ \\
Advanced asthma training (for example, asthma training course/ & $7(17 \cdot 9)$ & $461(41 \cdot 5)$ & $880(77 \cdot 8)$ \\
$\quad$ Royal College of General Practitioners asthma diploma course) & $8(20 \cdot 5)$ & $416(37 \cdot 5)$ & $378(33 \cdot 4)$ \\
Sitting in with a GP during a patient consultation with asthma & $2(5 \cdot 1)$ & $201(18 \cdot 1)$ & $325(28 \cdot 7)$ \\
Sitting in with a consultant in a hospital asthma or outpatient clinic & &
\end{tabular}


follow up of children. Objective monitoring of lung function was based upon peak flow measurement and few practices had, or used, spirometers.

The results of this survey seem to confirm that in responding practices much asthma care is now provided by practice nurses. The degree of involvement varies, and although most respondents seem to have undertaken delegated responsibility appropriate to their training, approximately a fifth of nurses running clinics by themselves had not undertaken formal training involving assessment. In view of the bias of the survey towards practices that are more aware of asthma this proportion is probably, higher elsewhere. The very significant role of the pharmaceutical industry in this training is also noted; such training does not normally entail evaluation and may be less likely to include continued contact between trainer and nurse.

Although this survey provides ample data about what is happening to a large number of people with asthma cared for within community asthma clinics, it does not provide answers regarding the effectiveness of that activity. One study of the effectiveness of a miniclinic for children showed only limited improvements in outcome, ${ }^{2}$ yet, another study disclosed appreciable improvements in several criteria of care using only checklists, booklets, and repeat audits without structured follow up and without a special asthma clinic. ${ }^{3}$ However, other small surveys have suggested beneficial outcomes from such clinics, ${ }^{4}$ and other methods of providing integrated care have been evaluated. ${ }^{6}$ We now need further controlled studies to evaluate the role and benefits of asthma clinics, and we need to know that they attract the patients "at risk" and whether clinics are the best, or only, way of providing care. These are essential questions if we are to be sure that we are organising effective high quality primary care for this common condition.

We thank our colleagues in primary care for their time completing our comprehensive questionnaire, QDP Ltd for its data processing skills, Ms Sue Theed of Market Ability who helped to evaluate the data, and Professor Sean Hilton and Dr Mark Levy for their constructive commer Dr Mark Levy for their constructive comments on the report. The costs of the survey were kindly borne by Allen and Hanburys, and administrative support was provided by the (he National Asthma Campaign.

1 Clark TJH, ed. The occurrence and cost of asthma. Worthing: Cambridge Medical Publications, 1990

2 Usherwood TP, Barber JH. Audit of process and outcome in a miniclinic for children with asthma. Fam Pract 1988;5:289-93.

3 Barritt PW, Staples EP. Measuring success in asthma care: a repeat audit. Brf Gen Pract 1991;41:232-6.

4 Charlton I, Charlton G, Broomfield J, Mullee MA. Audit of the effect of a nurse run asthma clinic on workload and patient morbidity in a general practice. $\mathrm{Br} \mathcal{F}$ Gen Pract 1991;41:227-31.

5 Charlton I, Charlton G, Broomfield J, Campbell M. An evaluation of a nurse run asthma clinic in general practice using an attitudes and morbidity questionnaire. Fam Pract 1992;9:154-60.

6 Grampian Asthma Study of Integrated Care (GRASSIC). Integrated care for asthma: a clinical, social, and economic evaluation. BMf 1994;308:559-64. 